\title{
Whole genome sequencing in the clinic: empowerment or too much information?
}

\author{
Amanda J. Berberich MD, Rosettia Ho BSc, Robert A. Hegele MD
}

- Cite as: CMAJ 2018 February 5;190:E124-5. doi: 10.1503/cmaj.180076

See related article at www.cmaj.ca/lookup/doi/10.1503/cmaj.171151

I $\mathrm{n}$ linked research, Reuter, Scherer and colleagues report on a pilot study of the Personal Genome Project Canada, a comprehensive open-source database that integrates genomic and health data. ${ }^{1}$ The authors performed whole genome sequencing of 56 healthy Canadian volunteers; this was the Canadian arm of the United Statesbased Personal Genome Project. ${ }^{2}$ Currently, outside the research context, clinical genetic tests typically assess a single gene or small gene panel related to a particular condition in an individual with a relevant personal or family history. But emerging next-generation sequencing technologies are beginning to enter the clinical space..$^{3-5}$

Next-generation sequencing encompasses several approaches: whole genome sequencing, whole exome sequencing and targeted sequencing. ${ }^{3-5}$ Whole genome sequencing reports all nucleotide bases in the entire genome, whole exome sequencing reports only the protein-coding regions (exons), and simpler targeted panels use next-generation sequencing chemistry to slice out information on predetermined disease-associated genes. The falling cost, increasing ease of application and comprehensive nature of whole genome sequencing will eventually make it the leading platform routinely used to define an individual's genetic profile.

The hundreds of thousands of variations detected by whole genome sequencing in an individual's DNA sample can have phenotypic consequences ranging from beneficial to neutral (predominantly) to potentially disease causing or disease associated. . $^{3-5}$ Adding knowledge of DNA variants to the family history could possibly affect diagnosis, prognostication or planning of therapeutic interventions, although this remains to be determined for most clinical scenarios. ${ }^{6-8}$ The greatest added value is seen when whole genome sequencing finds certain rare DNA variants of large effect size that completely underlie a disease phenotype, as in rare Mendelian single-gene disorders. ${ }^{7}$ In contrast, common DNA variants with small effects are associated with minor increases in risk of complex polygenic diseases, but because they are not directly causative, their clinical utility is less certain. ${ }^{7}$

In the last five years, the benefits of whole genome sequencing have been most apparent, through molecular genetic research, in the diagnosis of numerous novel genetic conditions. ${ }^{5,9}$ Whole genome and whole exome sequencing already have clear benefits in the diagnosis of children with rare Mendelian disorders. For instance, Canada's CARE for RARE consortium has shown repeatedly that early application of

\section{KEY POINTS}

- Whole exome and whole genome sequencing has already allowed for diagnosis of numerous novel genetic conditions and is changing the way we treat several cancers.

- The publication of early data from the Personal Genome Project Canada paves the way for future research that could allow whole genome sequencing to complement family history in the diagnosis and treatment of illness.

- Widespread use of whole genome sequencing could empower patients and doctors, but ethical concerns must be addressed carefully.

whole genome and whole exome sequencing can reduce the costly and psychologically burdensome diagnostic odyssey, which was previously endured by more than $50 \%$ of patients and families with uncharacterized or atypical rare diseases. ${ }^{9}$ Also, whole genome sequencing is helping to map the burden of somatically acquired variants in tumour tissues; this is shifting the concept of cancer treatment from one based on anatomic site or histology to one that considers the molecular genetic features of the tumour. Now, the question arises of whether increasingly affordable and available whole genome sequencing has utility in broader patient populations, such as generally healthy adults who might carry genetic susceptibility to later-onset diseases.

In the linked study, the authors identified tens of thousands of single nucleotide variants, small insertion or deletion variants, copynumber variants, larger structural variants and mitochondrial DNA variants in each of the initial 56 participants in Personal Genome Project Canada. Strict criteria for pathogenicity were applied to each variant, ${ }^{10}$ prioritizing them into a short list of likely disease-causing variants for each participant. They found that $25 \%$ of participants carried medically relevant genetic variants, with possible immediate health management implications, including six "pathogenic" or "likely pathogenic" variants and seven risk variants for cancer or common chronic diseases. Furthermore, almost all participants had other medically actionable findings, including recessive variants that conferred carrier status (e.g., for cystic fibrosis) and pharmacogenetic variants that underlay atypical responses to dozens of commonly used medications. Such results are more comprehensive and 
definitive than those offered by direct-to-consumer genetic testing services, which use older technology and detect only a tiny fraction of the genomic variation uncovered with whole genome sequencing.

The research is important for several reasons. It is important for a country as uniquely diverse as ours to develop and apply leadingedge made-in-Canada knowledge, methods, tools, databases and approaches to human genetics for our health system, in addition to simply importing these from other jurisdictions. The authors of the linked paper can trace their academic lineage through Canadian genetics hall-of-fame greats, and they continue this tradition today.

The framework is now established to evaluate clinical outcomes related to the application of whole genome sequencing in Canadian health care. The granddaddy of all whole genome evaluations is the family history. Does whole genome sequencing add any value to simple family history-taking? How many individuals would need to be screened with whole genome sequencing in order to prevent one clinically negative outcome? Answering such questions will require large, diverse cohorts of Canadians, studied in a range of health care settings, prospectively over a long period of time.

The imminent broadened application of whole genome sequencing data should hasten research to clarify the pathogenicity of DNA variants. There are negative implications if the risk associated with a variant is incorrectly attributed. ${ }^{6}$ Reuter and colleagues found that none of the "likely pathogenic" mutations identified among the participants was associated with clinically manifest disease at the time of the genetic diagnosis. In the absence of clinical manifestations, and without guidelines or directions on how to deal clinically with such information, disclosure of such genetic information to the participants and their health care providers could potentially have both positive and negative consequences. Underestimating the risk could result in false reassurance, but overestimating it could create needless worry.

The implications of the acquisition of whole genome sequencing data for consumers of health information - i.e., patients and the public - are not yet clear. ${ }^{6,7}$ Assuming science can minimize the uncertainty of results, what is the best means of delivering information from whole genome sequencing to patients? Should this be the bailiwick of primary care practitioners, medical subspecialists, genetic counsellors, nurse educators or lay groups, or some combination of these? Do different genetic conditions - e.g., monogenic versus polygenic - require different levels of expertise when translating findings? Is there a role for social media, artificial intelligence or virtual reality in the education of providers, patients or communities?

Finally, what about ethical, legal and social implications? Canada's recently passed Genetic Non-Discrimination Act (Bill S-201) protects individuals from genetic discrimination in the workplace, but information from whole genome sequencing could still potentially affect ability to obtain insurance, or might result in higher premiums. Also, what is the social cost of identifying variants that predispose an individual to conditions that carry stigma, such as certain neurobehavioural disorders, or those associated with certain ethnic ancestries?

The linked study highlights the potential of widespread implementation of whole genome sequencing, including early diagnosis and perhaps an opportunity to prevent or delay disease-associated outcomes or complications, screening of family members and implications for family planning. Yet, possible drawbacks include mis- diagnosis resulting from pathogenic misclassification, increased risk of incidental findings (too much information), diagnosing conditions for which nothing can be done, or increased use of resources triggered by a positive diagnosis. Although the potential negative aspects of whole genome sequencing warrant appropriate caution before we recommend its widespread use, the potential benefits deserve further careful and intensive evaluation, particularly within the Canadian context.

\section{References}

1. Reuter MS, Walker S, Thiruvahindrapuram B, et al. Personal Genome Project Canada: findings from the whole genome sequences of the inaugural 56 participants. CMAJ 2018;190:E126-36.

2. Ball MP, Bobe JR, Chou MF, et al. Harvard Personal Genome Project: lessons from participatory public research. Genome Med 2014;6:10.

3. Bick D, Dimmock D. Whole exome and whole genome sequencing. Curr Opin Pediatr 2011;23:594-600.

4. Farhan SM, Hegele RA. Genetics 101 for cardiologists: rare genetic variants and monogenic cardiovascular disease. Can J Cardiol 2013;29:18-22.

5. Boycott KM, Vanstone MR, Bulman DE, et al. Rare-disease genetics in the era of nextgeneration sequencing: discovery to translation. Nat Rev Genet 2013;14:681-91.

6. Manolio TA. Incorporating whole-genome sequencing into primary care: falling barriers and next steps. Ann Intern Med 2017;167:204-5.

7. Biesecker LG, Green RC. Diagnostic clinical genome and exome sequencing. $N$ Engl J Med 2014;370:2418-25.

8. Vassy JL, Christensen KD, Schonman EF, et al.; MedSeq Project. The impact of whole-genome sequencing on the primary care and outcomes of healthy adult patients: a pilot randomized trial. Ann Intern Med 2017;167:159-69.

9. Sawyer SL, Hartley T, Dyment DA, et al.; FORGE Canada Consortium; Care4Rare Canada Consortium. Utility of whole-exome sequencing for those near the end of the diagnostic odyssey: time to address gaps in care. Clin Genet 2016;89:275-84.

10. Richards S, Aziz N, Bale S, et al.; ACMG Laboratory Quality Assurance Committee. Standards and guidelines for the interpretation of sequence variants: a joint consensus recommendation of the American College of Medical Genetics and Genomics and the Association for Molecular Pathology. Genet Med 2015;17:405-24.

Competing interests: Robert Hegele has received honorariums for membership on advisory boards and speakers' bureaus for Akcea and Ionis, Aegerion Pharmaceuticals, Amgen, Gemphire Therapeutics, Regeneron Pharmaceuticals, Sanofi and Valeant, outside the submitted work. No other competing interests were declared.

This article was solicited and has not been peer reviewed.

Affiliation: Departments of Medicine and Biochemistry, and Robarts Research Institute, Schulich School of Medicine and Dentistry, Western University, London, Ont.

Contributors: All authors contributed to the conception and design of the work, drafted the manuscript, revised it critically for important intellectual content, gave final approval of the version to be published and agreed to be accountable for all aspects of the work.

Funding: Amanda Berberich is supported by the Clinical Investigator Program at the Schulich School of Medicine \& Dentistry, Western University, and by the Fernand Labrie Fellowship of the Canadian Society for Endocrinology. Rob Hegele is supported by the Jacob J. Wolfe Distinguished Medical Research Chair, the Edith Schulich Vinet Research Chair in Human Genetics, and the Martha G. Blackburn Chair in Cardiovascular Research. He has received operating grants from the Canadian Institutes of Health Research (Foundation Grant), the Heart and Stroke Foundation of Canada (G-15-0009214), and Genome Canada through Genome Quebec (award 4530).

Early release: Feb. 3, 2018

Correspondence to: Rob Hegele, hegele@robarts.ca 